\title{
Utilisation d'un réseau lamellaire multicouche comme monochromateur à bande passante étroite et comme polychromateur
}

S. Bac, G. Soullié*, A. Mirone**, M. Idir**, P. Guerin***, F.-R. Ladan*** et

P. Troussel

Commissariat à l'Énergie Atomique, Centre d'Études de Limeil-Valenton, 94195 Villeneuve-Saint-Georges cedex, France

* Laboratoire de Chimie-Physique, UA du CNRS 176, 11 rue Pierre et Marie Curie,

75231 Paris cedex 05, France

** Laboratoire de Spectroscopie Atomique et Ionique, Université Paris-Sud,

91405 Orsay cedex, France

*** Laboratoire de Microstructures et de Microélectronique, CNRS, 196 avenue

H. Ravera, 92220 Bagneux, France

Les réseaux lamellaires multicouches (RLM) combinent à la fois le bon pouvoir réflecteur des miroirs multicouches aux propriétés dispersives des réseaux. La structure de Bragg qui compose ces réseaux leur confère des propriétés qui peuvent être mises à profit pour utiliser de telles optiques comme monochromateur et comme polychromateur dans le domaine X-UV.

Les réseaux classiques fonctionnant en réflexion ne peuvent être utilisés, dans le domaine $\mathrm{X}-U V$, que sous incidence rasante. Pour s'affranchir d'une telle contrainte il est nécessaire d'adjoindre au réseau une structure de Bragg de manière à ce qu'il puisse être utilisé à toute incidence. Ces réseaux sont obtenus en gravant un miroir multicouche jusqu'à son substrat suivant des traits équidistants et de même largeur. En appelant $D$ et $d$ les périodes respectives du réseau et de la multicouche, il y a interférences constructives lorsque, pour une longueur d'onde donnée $\lambda$, les deux lois suivantes sont simultanément vérifiées :

$$
\cos \overline{0}-\cos \bar{\theta}_{0}=\frac{\mathrm{p} \lambda}{\mathrm{nD}}
$$

$$
\sin \bar{\theta}+\sin \bar{\theta}_{0}=\frac{m \lambda}{\text { nd }}
$$

où $\bar{\theta}_{0}$ et $\bar{\theta}$ sont respectivement l'angle d'attaque et l'angle d'observation définis entre le plan du réseau et les directions du rayonnement incident $\left(\bar{\theta}_{0}\right)$ et du rayonnement réfléchi $(\bar{\theta})$. p et $\mathrm{m}$ représentent respectivement les ordres du réseau et les ordres de Bragg de la multicouche. $\mathrm{n}$ est l'indice optique moyen du milieu diffuseur. La loi (1) est communément appelé loi des réseau, la seconde représente la loi de Bragg. Sous cette forme cette dernière tient compte de la propriété dispersive des réseaux. En combinant ces deux lois on obtient, pour une longueur d'onde fixée, la condition angulaire pour qu'il y ait interférences constructive :

$$
\bar{\theta}_{01}(\mathrm{p})=\operatorname{artg}\left(\frac{\mathrm{pd}}{\mathrm{mD}}\right)+\frac{1}{2} \operatorname{acs}\left(1-\frac{1}{2}\left[\left(\frac{\mathrm{m} \lambda}{\mathrm{nd}}\right)^{2}+\left(\frac{\mathrm{p} \lambda}{\mathrm{nD}}\right)^{2}\right]\right)
$$

Inversement, la relation donnant, pour une incidence donnée, la longueur d'onde diffractée dans un ordre $p$ du réseau est de la forme :

$$
\lambda(p)=\frac{2 m \sqrt{n^{2}-\cos ^{2} \theta_{0}}-\frac{2 p \cos \theta_{0}}{D}}{d}
$$

Article published by EDP Sciences and available at htD: $: /$ www. annphys.org or http://dx.doi.org/10.1051/anphys/1997031 
La loi (3) stipule qu'à une longueur d'onde donnée, chaque ordre du réseau possède un angle particulier, son angle de Bragg, pour lequel il a un maximum d'intensité [1, 2]. La loi (4) montre quant à elle qu'il est possible de séparer certaines longueurs d'onde d'un rayonnement polychromatique attaquant le réseau sous l'incidence $\theta_{0}$. Cet angle correspond à l'angle de Bragg des différentes longueurs d'ondes diffractées, chacune d'entre elles, dans un ordre $p$ particulier du réseau. On remarque qu'une telle optique fonctionne à l'instar d'un polychromateur [3].

Une autre particularité de ces optiques concerne leur sélectivité. En effet, la gravure permet de réduire considérablement la bande passante de la multicouche et ce, sans perte d'efficacité [4]. Plus le rapport cyclique $\Gamma$ du réseau (partie non gravée sur la période $D$ ) est petit, plus la bande passante est étroite. En fait la gravure a pour effet d'introduire des zones de vide dans le milieu diffuseur réduisant par conséquent le coefficient d'absorption moyen du milieu diffuseur mais aussi, le coefficient de réflexion aux interfaces. Plus pénétrant, le rayonnement atteint plus facilement les couches profondes ce qui permet de prévoir un nombre de paires de couches plus important pour le RLM. Le nombre d'éléments diffusants (interfaces) étant plus important pour le RLM par rapport au miroir multicouche, permet d'obtenir une bande passante plus étroite. La perte d'efficacité à chaque interface est globalement compensée par le nombre plus important de celles-ci chez le RLM.

Les courbes expérimentales suivantes illustrent chacun de ces faits.

figure a : miroir multicouche

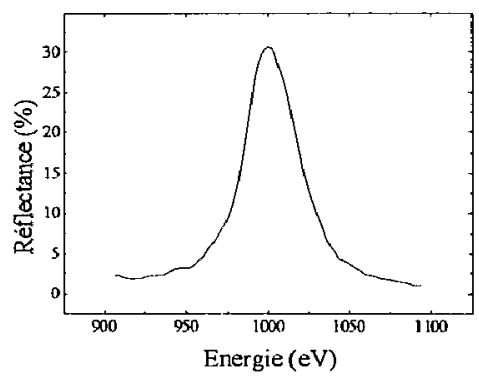

figures a, b, c : multicouche W/C 100 paires, $d=41.2 \AA$. Pas de gravure $\mathrm{D}=1 \mu \mathrm{m}$, rapport cyclique $\Gamma=0.1$.

figure a : courbe d'efficacité de la multicouche.

figure b: courbe d'efficacité du RLM. On remarque le rétrécissement de la bande passante (facteur 1/4) par rapport à la multicouche.

figure $c$ : spectre de diffraction en u.a $=f(\theta)$. Séparation angulaire et en énergie du rayonnement synchrotron Super A.C.O. par le RLM. L'angle d'attaque choisi est l'angle de Bragg à $1 \mathrm{keV}$ de l"ordre $\mathrm{p}=+1$. La longueur d'onde portée par chaque pic se déduit à partir de la relation (4). Le RLM diffracte les énergies dans les trois ordres $m=1,2,3$ de la multicouche.

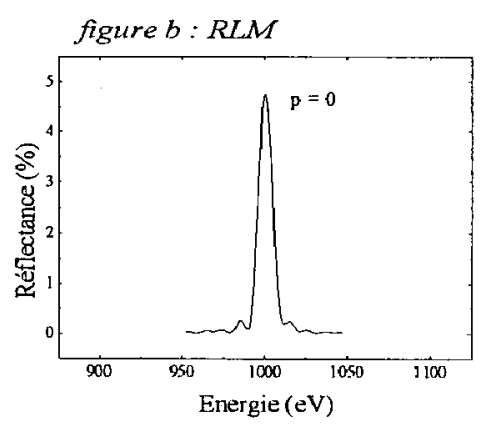

figure $c: R L M$ "polychrornateur"

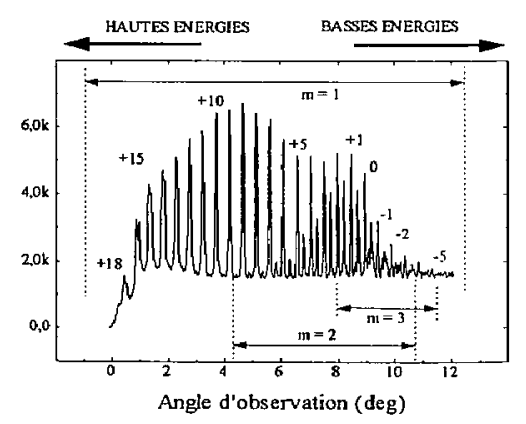

\section{Références :}

[1] A. E. Sammar, Thèse de l'université Pierre et Marie Curie Mai 1993

[2] S. Bac and al, Journal of X-ray Science and Technology 5, 161-180 (1995).

[3] S. Bac, Thèse de l'université Pierre et Marie Curie 11 Juillet 1994

[4] A. E. Sammar and al, C. R. Acad. Sci. Paris. T. 316. Série II. P. 1-0, 1993 